\title{
Odontoblastic Cell Quantification and Apoptosis within Pulp of Deciduous Teeth Versus Pulp of Permanent Teeth
}

\author{
Elena Bardellini*/ Francesca Amadori**/ Amerigo Santoro***/ Giulio Conti****/ Giovanna \\ Orsini*****/ Alessandra Majorana******
}

\begin{abstract}
Objective: While the odontoblast ability to respond to injury in permanent teeth (PT) is well established, there is a lack of knowledge about deciduous teeth (DT). Aim of this study was to compare the odontoblasts activity within the pulp of DT versus the pulp of PT. Study design :Dental pulp was obtained from forty-two $D T$ and twenty-seven PT extracted from sixty-five patients (aged 6-16 years). Histomorphometry was carried out and the quantification of odontoblastic layer was assessed. Dental pulps of DT and PT were stained for anti-ssDNA, BCL-2, BCL-x, BAX, caspase3. Results : Pulps from DT were characterized by reduction of odontoblastic layer and greater occurrence of apoptotic odontoblasts. Pro-apoptotic BAX phenotype expression on odontoblasts correlated with the occurrence of numerous activated caspase 3 odontoblasts in DT. The number of BAX positive cells was significantly higher compared to BCL-2 positive cells in the odontoblastic layer of the $D T(p=0.03)$. Since $B A X$ and $B C L-2$ proteins have an inverse role in the regulation of the apoptosis, this finding suggests that odontoblasts have a predominant pro-apoptotic phenotype in DT. Conclusion: According to our results, the odontoblasts of DT can be assumed to have a lower reparative activity if compared to odontoblasts of PT.
\end{abstract}

Key words: apoptosis; deciduous tooth; permanent tooth; odontoblast; pulp.

\section{INTRODUCTION}

$\mathrm{T}$ The dental pulp not only provides nutritional and sensory properties to dentin but also has its own reparative capacity. ${ }^{1-4}$ This potential has important implications for dental therapies. Dentinogenesis has been extensively studied to comprehend the development and mineralization of this connective tissue. The odontoblast ability to respond to injury (e.g. caries, cavity preparation) and up-regulate its secretory activity leading to deposition of reactionary

*Elena Bardellini,University of Brescia, School of Dentistry, Brescia, Italy.

**Francesca Amadori, University of Brescia, School of Dentistry,Brescia, Italy.

***Amerigo Santoro, Spedali Civili of Brescia, Department of Pathology Brescia, Italy.

**** Giulio Conti, University of Milan, Dental Clinic, Milan, Italy

*****Giovanna Orsini, Department of Clinical Sciences and Stomatology, Polytecnic University of Marche, Ancona, Italy.

******Alessandra Majorana,University of Brescia, School of Dentistry,Brescia, Italy.

Send all correspondence to:

Dr. Elena Bardellini

Dental Clinic

p.le Spedali Civili 1

25133 Brescia

Italy

Phone: 00390303995780

Fax. 0039030303194

E-mail: elena.bardellini@unibs.it dentin is well established. ${ }^{1,6-7}$ The important feature of this response is that there is no cell renewal and the odontoblasts have to survive the injury. The process of reactionary dentinogenesis is different from the reparative dentinogenesis, where the intensity of the injury is of a magnitude that results in odontoblast death and cell renewal by a new generation of odontoblasts-like cells, that may differentiate from progenitor cells within the pulp and secrete a reparative dentin matrix. ${ }^{1}$ In the case of the injury leading to pulpal exposure, this reparative dentinogenesis may give rise to dentin bridge formation.

Since reparative dentinogenesis may start after the elimination of damaged odontoblasts, it is noteworthy to understand the death regulation of odontoblasts.

The number of odontoblasts decline with age and the apoptosis, as programmed cell death, has been implicated in this biological process. ${ }^{8}$ There are several findings confirming that apoptotic cell death play a role during tooth development and in repair-related tooth remodelling such as injured pulp, in permanent teeth. ${ }^{9}$

However, there is a lack of knowledge in understanding the apoptotic mechanism in odontoblasts of deciduous teeth and their potential response to pulp injuries.

The purpose of the present study was to evaluate a series of deciduous (DT) and permanent teeth (PT) to clarify the odontoblasts physiological activity in deciduous teeth compared to permanent teeth. The hypotheses tested were that 1) there are morphological differences between the odontoblastic layer of deciduous and permanent teeth and 2) there is an apoptotic mechanism that regulates odontoblasts response, in primary dentition. 


\section{MATERIALS AND METHOD}

\section{Tissues}

Dental pulp was obtained from forty-two DT and twenty-seven PT extracted from sixty-five different patients between 2009 and 2012. All patients, including the parents of minor children, gave informed consent to the treatment procedure. The age of patients ranged from 6 to 16 years (mean 11 years), with a slight prevalence of males $(33 / 65,50.7 \%)$. DT and PT were extracted for orthodontic treatment $(n=39)$, extrusive trauma $(n=19)$, delayed permute $(n=11)$. The extracted DT were all in the second stage of development with well-defined roots. DT were extracted from patients aged 6-11 years while PT from patients aged 11-16 years. After extraction, all the teeth had been immediately fixed in 10\% formalin for $24-48$ hours. The enamel was removed with a diamond burr in order to minimize the thickness of the hard tissue and the specimens were successively decalcified in EDTA solution for 12 hours and embedded in paraffin.

\section{Histomorphometry}

Histomorphometry was carried out on photomicrographs of the entire section stained from each specimen by means of the Olympus BX-60 microscope, equipped with the Olympus DP-70 digital camera. Image analysis was performed by Analysis 3.2 soft imaging system GMBH. The quantification of odontoblastic layer extent was assessed by the odontoblastic index (OI), defined as the ratio of odontoblastic layer length to pulp chamber perimeter $\mathrm{x} 100$.

\section{Immunohistochemistry}

Dental pulps of six DT and twenty PT were stained for anti-ssDNA (Bender Med-Sysytems, Vienna, Austria, EU), a typical marker of apoptosis, using formalin-fixed tissue sections according to manufacturer's protocol. The apoptotic index (AI) was defined for odontoblastic layer as the ratio of ssDNA-positive odontoblast to all counted odontoblastic cells x 100. Sections from seventeen PT and twelve DT dental pulps were also immunostained for BCL-2 (dilution 1:50, Dako, Golstrup, Denmark, EU), BCL-x (dilution 1:50, Abcam, Cambridge, United Kingdom, EU), BAX (dilution 1:100, Abcam). Sections were also immunostained for activate caspase-3 (dilution 1:200, Trevigen, Gatthersburg, MD, USA). After heating (750W) for 15 min in EDTA buffer, an indirect immunoperoxidase technique (StrAviGen Multilink Kit, Biogenex, San Ramon, CA, USA) was used. Nuclei were counterstained with Haematoxylin. For each case, the number of positive cells was counted on photomicrographs from two randomly selected high power fields (40x objective, 10x ocular), corresponding to $0.07 \mathrm{~mm}^{2}$ of tissue, using an Olympus BX60 microscope. Positive cells were ascertained on the basis of a cytoplasmic staining. In addition, only activated caspase- $3^{+}$and $\mathrm{ssDNA}^{+}$cells were also identified on the basis of nuclear labelling. Sections represented positive controls derived from cases of reactive lymphadenitis, Hodgkin's lymphoma, normal prostatic tissue, respectively for BCL-2, BCL-x, BAX antigens. Positive controls for activated caspase- 3 and ssDNA were represented by sections from Burkitt's lymphoma. The omission of the primary antibody or the use of an isotype matched, irrelevant antibody, represented negative controls.

\section{Statistical analysis}

Statistical analysis was performed by using the Mann-Whitney rank- $U$ test for quantitative data (differences between DT and PT) and Fisher's exact test for odontoblastic layer presence. The Wilcoxon rank test was used to analyze BAX and BCL-2 expression on odontoblasts from DT. The Pearson's correlation coefficient was calculated to determine the correlation between the number of positively stained cell respectively for BAX and activated caspase-3. Statistical significance was defined as $\mathrm{p}<0.05$.

\section{RESULTS}

Quantitative data are illustrated in Table 1. Dental pulps from DT were characterized by reduction of odontoblastic layer and occurrence of apoptotic odontoblasts (Figure 1). The observation of odontoblastic layer in the sections of dental pulp showed that odontoblasts were significantly reduced in twenty-one out of forty-two (50\%) deciduous teeth (DT) if compared to four out of twenty-seven (14.8\%) permanent teeth (PT) $(p=0.04)$. To evaluate whether apoptosis may be involved, a sensitive analysis, using ssDna immunostaining in deciduous and permanent dental pulps was applied. SsDna ${ }^{+}$cells were observed in the majority of deciduous dental pulps $(66.6 \%)$ in contrast to permanent dental pulps $(45 \%)$ and appeared as single scattered cells located to the odontoblastic layer. Furthermore, the apoptotic index (AI) was quantified in odontoblastic cells and, even if the differences were not significant, it was higher in deciduous dental pulps compared to the pulps of PT $(7.80 \pm 4.13$ vs $4.70 \pm 1.68)$.

Odontoblastic index (OI) was significantly lower in DT as compared to PT. Histomorphometric results showed that odontoblastic layer length was lower in DT compared to PT $(10.12 \pm 0.81$ vs 12.74 \pm 1.04$)$. The odontoblastic index (OI) was significantly lower in DT compared to PT $(30.15 \pm 5.00 v s 51.81 \pm 6.97, p=0.03)$.

Pro-apoptotic BAX phenotype expression on odontoblasts correlated with the occurrence of numerous activated caspase- $3^{+}$ odontoblasts in DT $(\mathrm{p}=0.01)$.

Immunostaining for the anti-apoptotic BCL-2 and BCL-X proteins (Figure 2 and Figure 3) and the pro-apoptotic BAX protein (Figure 4) was performed in sections obtained from DT and PT. Table 1 exhibits the results of immunohistochemistry. No or rare odontoblasts showed positivity for BCL-x protein in DT and PT. On the other hand, BCL-2 protein was detected in odontoblasts and scattered fibroblasts and lymphocytes, when these were present. In particular, BCL-2 protein was expressed in $33.3 \%$ of DT and counting analysis showed that number of BCL-2 ${ }^{+}$odontoblasts was slightly higher in DT compared to PT $(2.75 \pm 1.60 v \mathrm{~s}$ $2.51 \pm 1.60)$ even if the difference was not statistically significant $(p=0.140)$. BAX protein was detected in odontoblasts, fibroblasts and in rare lymphocytes, if these were present. BAX protein was expressed on odontoblasts in $83.3 \%$ of DT and in $40.7 \%$ of PT . Moreover, high number of $\mathrm{BAX}^{+}$odontoblasts was identified in DT in contrast to PT, and the difference was statistically significant $(24.79 \pm 11.93$ vs $4.27 \pm 1.48, p<0.01)$. Since BAX and BCL-2 proteins have an inverse role in the regulation of the apoptosis, it was interesting to find that number of $\mathrm{BAX}^{+}$cells was significantly higher compared to $\mathrm{BCL}-2^{+}$cells in the odontoblastic layer of the DT $(24.79 \pm 11.93$ vs $2.75 \pm 1.60, p=0.03)$, suggesting that odontoblasts have a predominant pro-apoptotic phenotype in DT. 
These results were corroborated by detection of nuclear and cytoplasmic activated caspase $3^{+}$, a marker of commitment to apoptosis, on numerous odontoblasts in DT $(20.37 \pm 5.77)$ (Figure 5). Finally, statistically analysis revealed strong linear correlation between numbers of $\mathrm{BAX}+$ and activated caspase-3+ odontoblasts in DT $(\mathrm{r}=0.908, \mathrm{p}=0.01)$.
Table 1. Extent of odontoblastic layer as determined by odontoblastic index (OI) and odontoblastic cells, expressing different markers (BCL-2,BCL-x, BAX, CASP-3A), related to apoptosis, in deciduous (DT) and permanent (PT) teeth. Values are expressed as the mean number and standard deviation (SD).

\begin{tabular}{lccc}
\hline & $\begin{array}{c}\text { DT } \\
\text { mean } \pm S D\end{array}$ & $\begin{array}{c}\text { PT } \\
\text { mean } \pm S D\end{array}$ & p value \\
\hline $\begin{array}{lccc}\text { Odontoblastic Index } \\
\text { (OI) }\end{array}$ & $30.15 \pm 5.00$ & $51,81 \pm 6.97$ & $\mathrm{p}<0.05$ \\
BCL-2 & $2.75 \pm 1.60$ & $0.00 \pm 0.00$ & -- \\
BCL-x & $0.00 \pm 0.00$ & $0.70 \pm 0.42$ & -- \\
BAX & $24.79 \pm 11.93$ & $4.27 \pm 1.48$ & $\mathrm{p}<0.01$ \\
CASP-3A & $20.37 \pm 5.77$ & $3.48 \pm 3.61$ & $\mathrm{p}<0.01$ \\
\hline
\end{tabular}

Fig. 1 Coronal (a) and radicular (c) histological sections of a deciduous tooth: it's showed lower cellular density of the odontoblasts (Od, arrowheads), compared to the coronal (b) and radicular (d) sections of a permanent tooth. D: dentin; dP: deciduous dental pulp; pP: permanent dental pulp (Magnification 100x)

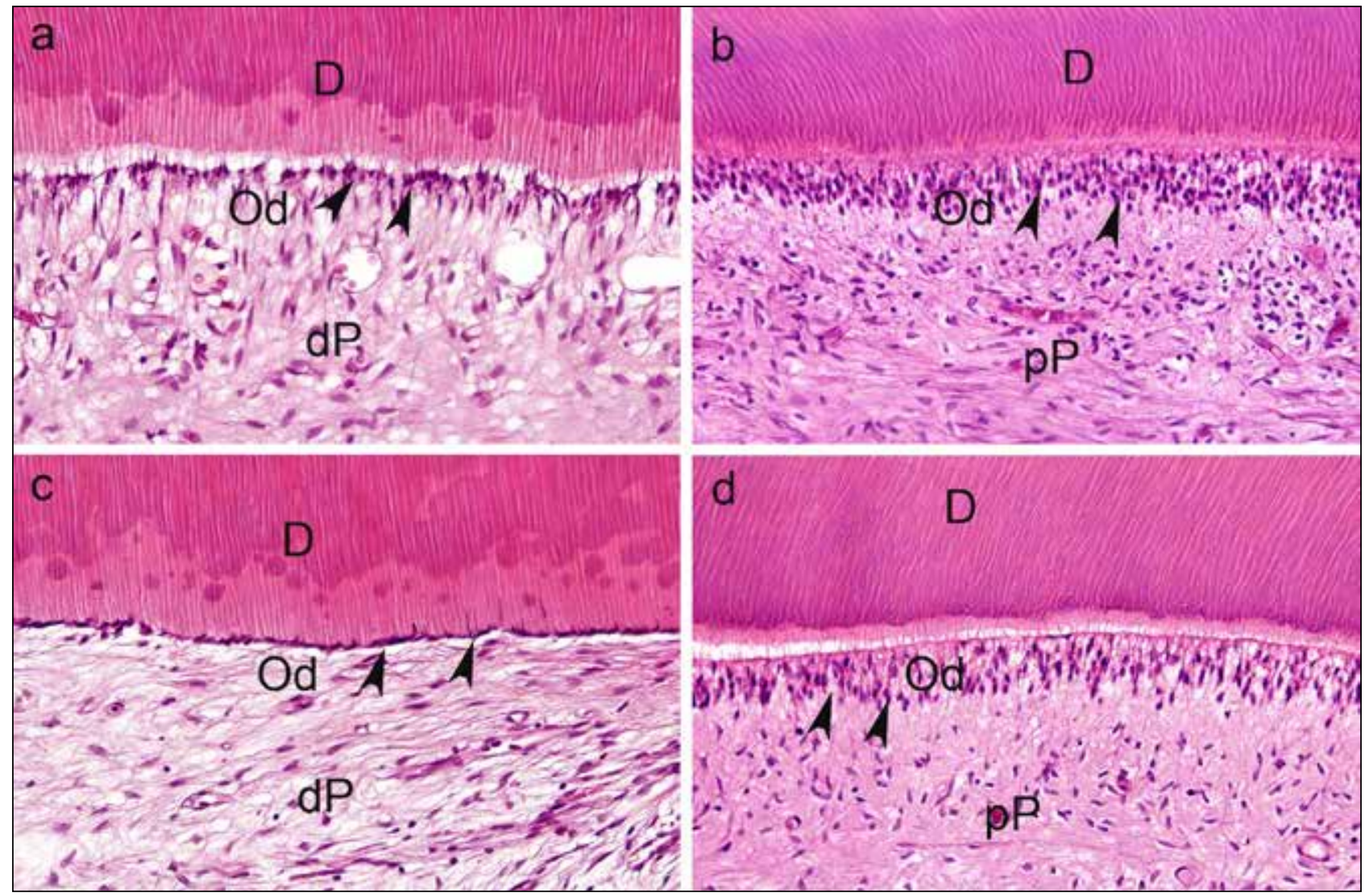

Fig. 2 Expression of BCL-2 in the odontoblasts of deciduous (a, b) and permanent (c) dentition: immunohistochemistry showed low BCL-2 positivity only in some deciduous teeth (b). Od: odontoblasts (arrowheads); D: dentin; dP: deciduous pulp; pP: permanent pulp (Magnification 200x)

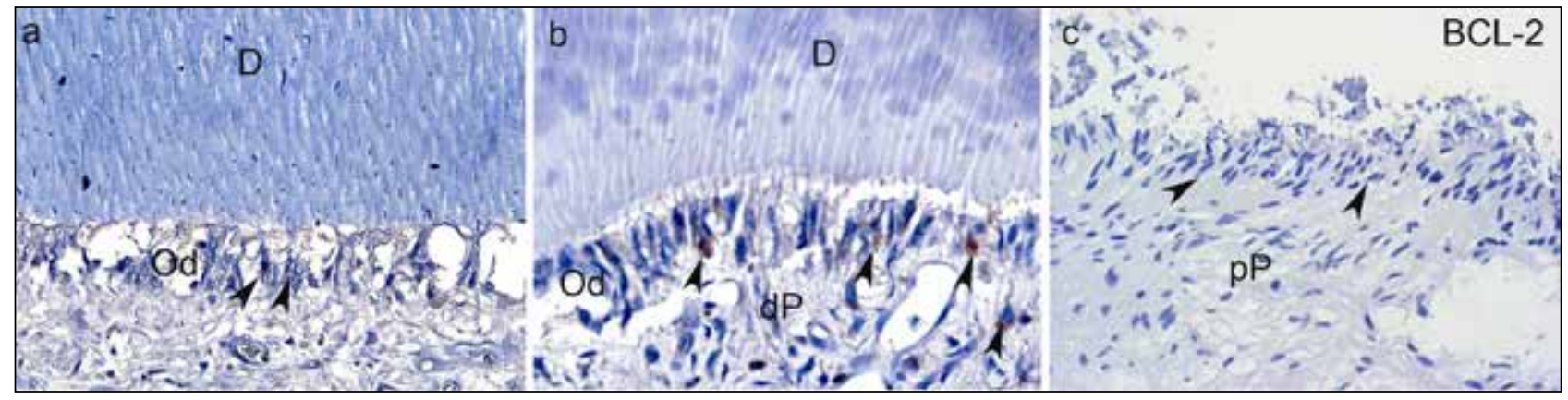


Fig. 3 Immunohistochemistry showed BCL-x negativity in both deciduous (a) and permanent (b) dentition. Od: odontoblasts (arrowheads); D: dentin; dP: deciduous pulp; pP: permanent pulp (Magnification 200x)

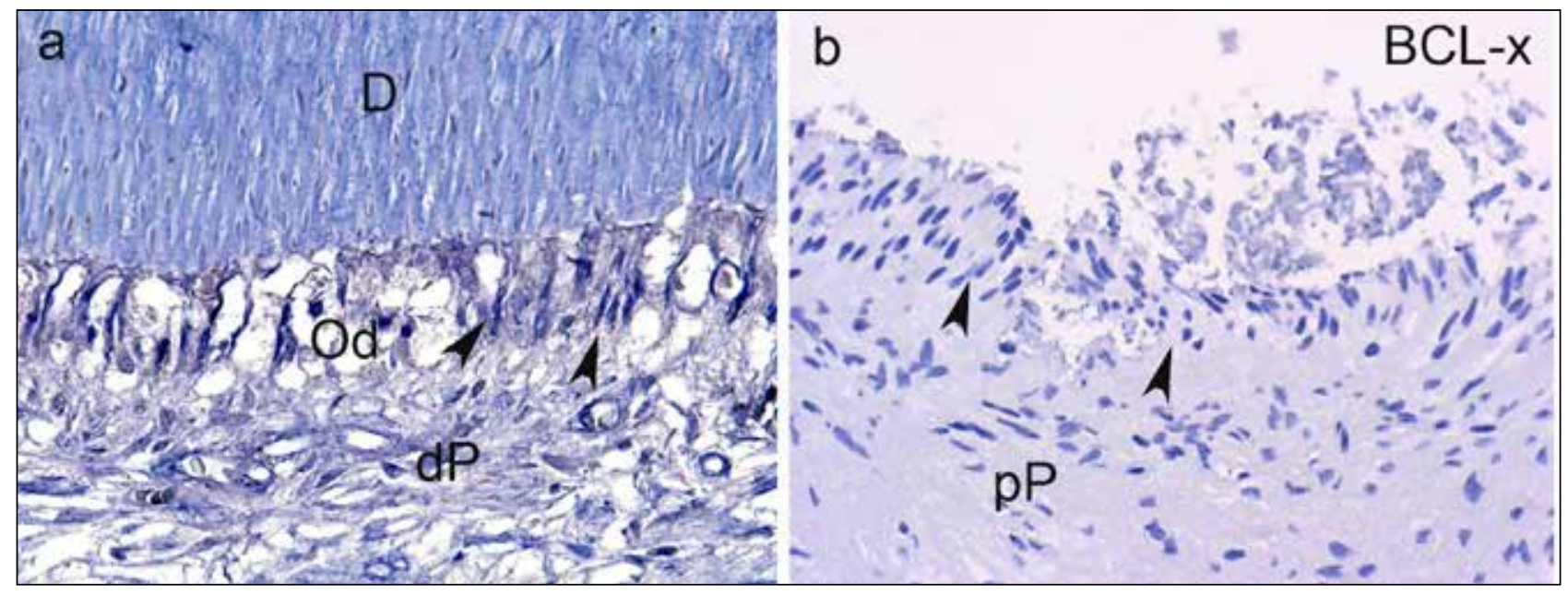

Fig. 4 Immunohistochemistry showed the cytoplasmic and nuclear expression of BAX in odontoblasts (Od, arrowheads) laying the pulp of deciduous (dP) teeth (a), whereas no BAX expression was detected on histological sections obtained from permanent dentition (b). D: dentin; pP: permanent pulp (Magnification 200x)

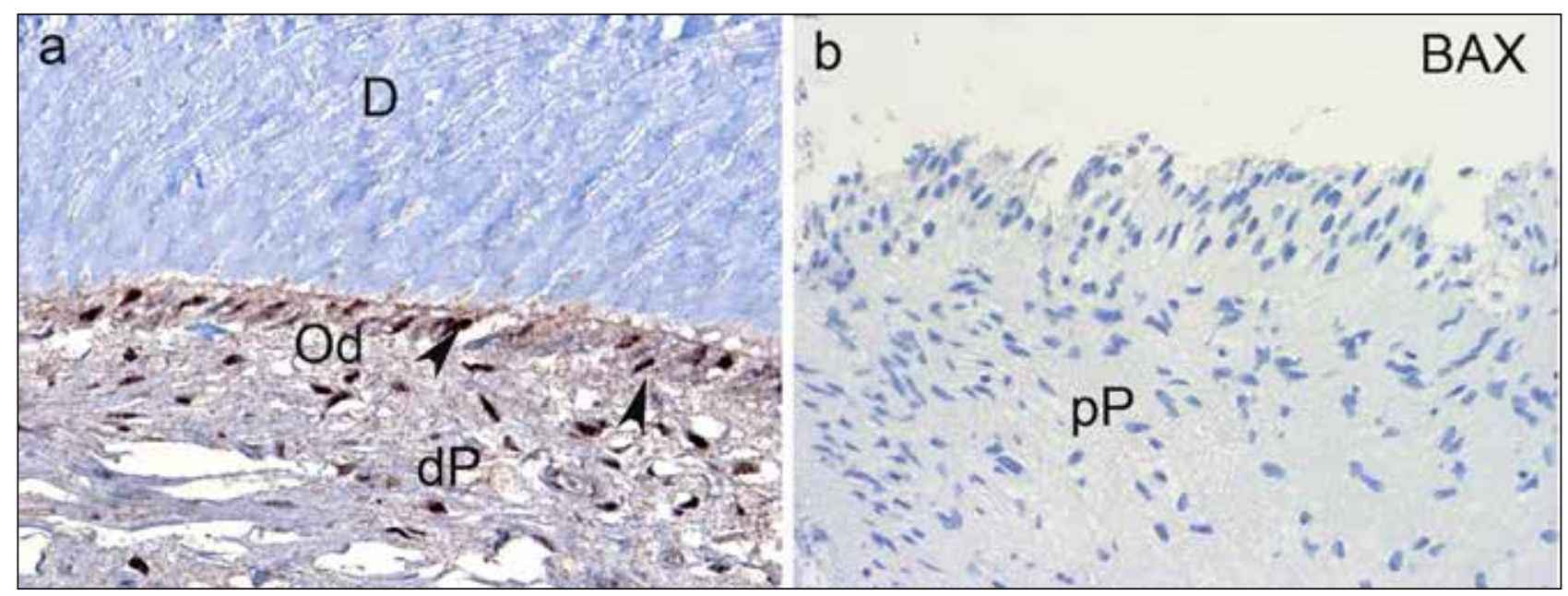

Fig. 5 Immunohistochemistry showed positive expression of activated caspase-3 (casp-3) in histological sections of deciduous dentition. An intense (a) or moderate (b) labelling was observed on the cytoplasms, nuclei and odontoblastic processes of the odontoblasts (Od, arrowheads) laying the deciduous dental pulp (dP). D: dentin (Magnification 200x)

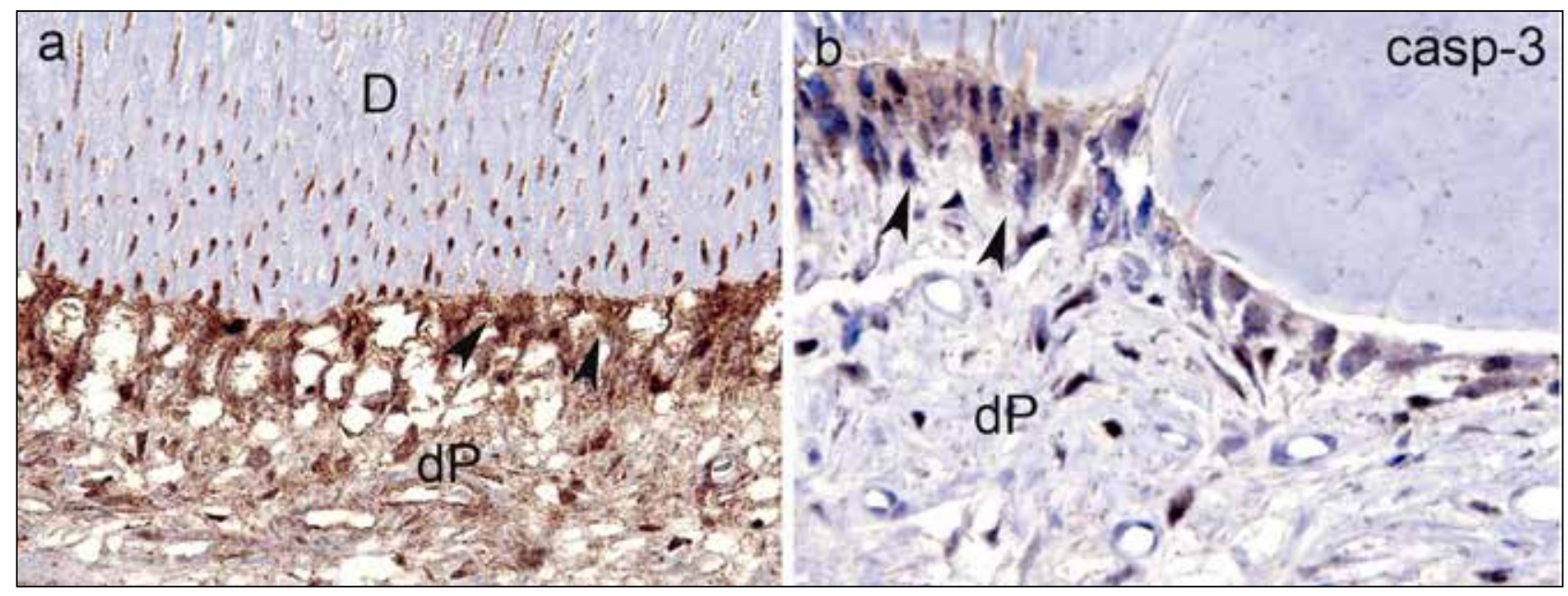




\section{DISCUSSION}

The results of the present study support the two research hypotheses, showing that: 1) there is a morphological apparent reduction of the odontoblastic layer in dental pulps of DT compared to PT, and 2) there is greater occurrence of apoptotic odontoblasts in DT compared to PT. It can be speculated that the mode of odontoblasts deficiency in dental pulps of DT is apoptosis, in accordance with previous studies demonstrating that odontoblasts decrease during their life-cycle by apoptotic cell death. ${ }^{8,9}$

Apoptosis is defined as an active process of cell death that ultimately is mediated by activated caspase- 3 and consequently activation of downstream DNAses. ${ }^{10,11}$ Indeed, previous immunohistochemical reports demonstrated that apoptosis plays a crucial role not only during embryonic development, but also in the maintenance of dental tissue homeostasis, by eliminating cells that have already achieved their genetic program, thus controlling the pattern, shape and size of the teeth. ${ }^{12-14}$

A close correlation exists between the volume of the dental pulp chamber and the age of the teeth, because there is a continuous dentin deposition that decreases the volume occupied by the odontoblast/dentin interface. Only one report described this physiological event, showing that massive odontoblast apoptosis occurs during a 4-year period. ${ }^{8}$ Some studies evaluated apoptosis in the odontoblastic layer of intact and injured permanent human teeth. ${ }^{15,16}$ However, to the authors knowledge, there are no studies evaluating the odontoblast presence (by means of odontoblastic index) and apoptosis (by means of apoptotic markers such as BAX, BCL-2 and Caspase-3), both in DT and in PT.

Indeed, the morphological observations of the present study may lead to hypothesise a real difference in odontoblast activity between DT and PT. Histomorphometry and immunohistochemistry data seem to confirm this hypothesis. By means of OI computation, analysis of odontoblastic layer length suggests that the difference of odontoblasts between DT and PT is significantly high. This finding allows evaluating the occurrence of a pro-apoptotic phenotype expression on odontoblasts, using in situ immunohistochemistry for apoptotic regulatory BAX, BCL-2 and BCL-X proteins. Both BAX and BCL-2 have been expressed on odontoblasts, but a significative difference between DT and PT has been documented only for BAX expression. Indeed, while PT displayed scattered $\mathrm{BAX}^{+}$ odontoblasts, DT showed higher numbers of $\mathrm{BAX}^{+}$odontoblastic cells. Furthermore, these findings are associated with a significant prevalence of pro-apoptotic BAX protein over anti-apoptotic BCL-2 protein in odontoblastic cell population from DT, revealing the occurrence of pro-apoptotic phenotype on these cells. Since it has been demonstrated that high $\mathrm{BAX} / \mathrm{BCL}-2$ ratio determines the cell susceptibility to apoptosis following trigger signals, we have tried to detect activated caspase-3, one of the key cystein protease of apoptosis. ${ }^{17}$ Caspase- 3 is involved in the proteolytic cleavage of key downstream proteins, such as poly(ADP-ribose) polymerase (PARP), which ultimately results in DNA fragmentation and apoptotic cell death.

Findings showed that the occurrence of pro-apoptotic phenotype on odontoblasts of DT might be correlated with the degree of caspase-3 activation, a marker of commitment to apoptosis. ${ }^{18}$ In fact, a significant increased number of $\mathrm{BAX}^{+}$odontoblasts were found in DT, as opposed to PT, and they were associated with activation of caspase-3, consequently being committed to apoptosis. These findings may corroborate the hypothesis that apoptotic cell death is responsible for the quantitative difference of odontoblasts evidenced by histomorphometric changes in DT. Odontoblasts are highly differentiated post-mitotic cells, which synthesize the circumpulpal dentin and lay down physiological secondary dentin. 19 The decrease in this cellular activity may influence the tooth development and may change the response to tissue damage, since tertiary dentin is deposited beneath the site of injury by existing odontoblasts. ${ }^{15,20}$ According to the present study, the odontoblasts of DT can be assumed to have a lower reparative activity if compared to odontoblasts of PT. Many reports have documented that the vital pulp treatments used in primary dentition have lower success rates in comparison to permanent dentition but this fact has been no correlated with the extent of odontoblastic layer and the apoptosis of odontoblastic cell population. ${ }^{17,21-24}$ Further studies are needed to evaluate whether this clinical correlation might be confirmed.

\section{CONCLUSION}

According to the present study, the odontoblasts of DT can be assumed to have a lower reparative activity if compared to odontoblasts of PT. Many reports have documented that the vital pulp treatments used in primary dentition have lower success rates in comparison to permanent dentition but this fact has been no correlated with the extent of odontoblastic layer and the apoptosis of odontoblastic cell population. ${ }^{17,21-24}$ Further studies are needed to evaluate whether this clinical correlation might be confirmed. 


\section{REFERENCES}

1. Goldberg M, Smith AJ. Cells and extracellular matrices of dentin and pulp: a biological basis for repair and tissue engineering. Crit Rev Oral Biol Med 15(1): 13-27, 2004.

2. Lesot H, Bègue-Kirn C, Kübler MD, Meyer JM, Smith AJ, Cassidy N, Ruch JV. Experimental induction of odontoblast differentiation and stimulation during reparative processes. Cell Mater 3: 201-217, 1993.

3. Smith AJ, Lesot H. Induction and regulation of crown dentinogenesis: embryonic events as a template for dental tissue repair?. Crit Rev Oral Biol Med 12:425-437, 2001.

4. Linde A, Goldberg M. Dentinogenesis. Crit Rev Oral Biol Med 4:679$725,1993$.

5. Ruch JV, Lesot H, Bègue-Kirn C. Odontoblast differentiation. Int J Develop Biol 39: 51-68, 1995.

6. Smith AJ, Tobias RS, Cassidy N, Plant CG, Browne RM, Bègue-Kirn C et al. Odontoblast stimulation in ferrets by dentine matrix components. Archives of Oral Biology 39;13-22, 1994.

7. Smith AJ, Cassidy N, Perry H, Bègue-Kirn C, Ruch Jv, Lesot H. Reactionary dentinogenesis. Int J Develop Biol 39:273-280, 1995.

8. Franquin JC, Remusat M, Abou Hashieh I, Dejou J. Immunocytochemical detection of apoptosis in human odontoblasts. Eur J Oral Sci 106 Suppl 1: 384-387, 1998.

9. Mitsiadis TA, De Bari C, About I. Apoptosis in developmental and repair-related human tooth remodeling: a view from the inside. Exp Cell Res 314(4): 869-877, 2008.

10. Kerr JF, Wyllie AH, Currie AR. Apoptosis: a basic biological phenomenon with wide-ranging implications in tissue kinetics. Brit J Cancer 26: 239-257, 1972.

11. Nunez G, Benedict Ma, Hu Y, Inohara N. Caspases: the proteases of the apoptotic pathway. Oncogene 17: 3237-3245, 1998.

12. Vaahtokari A, Aberg T, Thesleff I. Apoptosis in the developing tooth: association with an embryonic signaling center and suppression by EGF and FGF-4. Develop 122: 121-129, 1996.
13. Kim JY, Cha YG, Cho SW, Kim EJ, Lee MJ, Lee JM et al. Inhibition of apoptosis in early tooth development alters tooth shape and size. J Dent Res 85(6):530-535, 2006.

14. Jernvall J, Aberg T, Kettunen P, Keränen S, Thesleff I. The life history of an embryonic signaling center: BMP-4 induces p21 and is associated with apoptosis in the mouse tooth enamel knot. Develop 125(2):161-169, 1998.

15. Larmas M. Odontoblast function seen as the response of dentinal tissue to dental caries. Adv Dent Res15: 68-71, 2001.

16. Larmas M, Sándor GK. Enzymes, dentinogenesis and dental caries: a literature review. J Oral Maxillofac Res 29; 5(4):e3, 2014. doi: 10.5037/ jomr.2014.5403

17. Oltvai ZN, Milliman CL, Korsmeyer SJ. Bcl-2 heterodimerizes in vivo with a conserved homolog, Bax, that accelerates programmed cell death Cell 74: 609-619, 1993.

18. Mazumder S, Plesca D, Almasan A. Caspase-3 activation is a critical determinant of genotoxic stress-induced apoptosis. Meth Molec Biol 414: 13-21, 2008.

19. Lesot H, Lisi S, Peterkova R, Peterka M, Mitolo V, Ruch JV. Epigenetic signals during odontoblast differentiation. Adv Dent Res 15: 8-13, 2001.

20. Thesleff I, Keranen S, Jernvall J. Enamel knots as signaling centers linking tooth morphogenesis and odontoblast differentiation. Adv Dent Res 15: 14-18, 2001.

21. Fuks AB. Current concepts in vital primary pulp therapy. Eur J Paed Dent 3: 115-120, 2002.

22. Fuks AB. Vital pulp therapy with new materials for primary teeth: new directions and Treatment perspectives. Ped Dent 30: 211-219, 2008.

23. Kopel HM. Considerations for the direct pulp capping procedure in primary teeth: a review of the literature. ASDC J Dent Child 59: 141-149, 1992.

24. Ranly DM, Garcia-Godoy F. Current and potential pulp therapies for primary and young permanent teeth. J Dent 28: 153-161, 2000. 